ANTIMICROBIALS

\section{Computational culture}

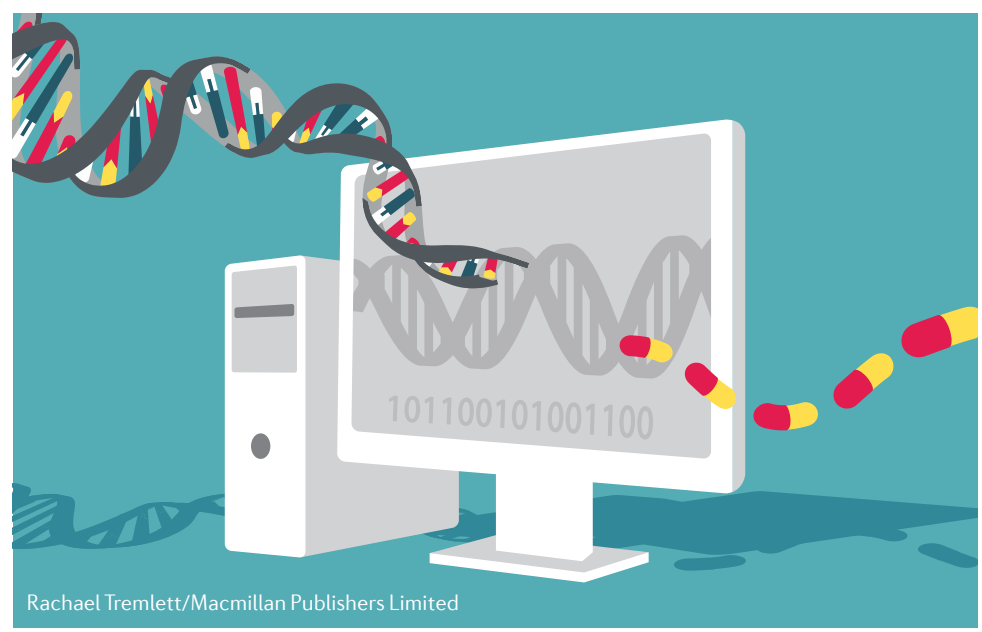

Evolutionary pressure means that new antimicrobial drugs are in constant demand, a problem compounded by treatments being overprescribed by doctors and not completed by patients. Most antimicrobial drugs in use today are natural products or synthetic and semi-synthetic analogues. The discovery of bioactive natural products has traditionally begun with culturing bacteria and then extracting and isolating the active components. Now, Sean Brady and co-workers have used existing bioinformatic tools to identify what they term syntheticbioinformatic natural products.

"The traditional approach to natural products drug discovery has some potential flaws," explains Brady. "A key issue is that most bacteria do not readily grow in cultures, and even when they do grow, they do not express all their potential secondary metabolites." Brady and his team avoid these problems by combining high-throughput DNA sequencing with bioinformatic algorithms predicting the chemical products encoded by the gene clusters. With the structures of these products in hand, they then use solid-phase peptide synthesis to produce the compounds for testing. Using this approach, they have identified two potential new drugs - an antifungal agent and an antibiotic.

Starting with around 3,000 complete bacterial genomes obtained from the National Center for Biotechnology Information (NCBI), Brady and co-workers used known algorithms to identify biosynthetic gene clusters and to predict the structures of the nonribosomal peptides that they encode. After narrowing the pool of gene clusters by eliminating some that would be problematic for rapid automated synthesis, they selected those that contained either a starter condensation domain or a thioesterase domain - that is, those that begin or end the natural product synthetic sequences. Their prediction of peptide sequences was imperfect, and from 280 gene clusters that fit the above criteria they were able to generate 288 peptide sequences of interest for testing.

The peptides were then synthesized in 96-well plates using Fmoc-based solid-phase peptide synthesis.

Screening for bioactivity against the fungal pathogen Candida albicans and the ESKAPE pathogens (which are known to cause most hospitalacquired infections in the United States) resulted in six hits, two of which were selected for detailed analysis.

The first peptide identified, a 13-mer, contains $7 \mathrm{D}$-amino acids and 3 non-proteinogenic residues. The gene cluster that encodes it comes from Paenibacillus mucilaginosus and is similar to the one that encodes the known tridecaptin antibiotics. Notably, the bacterium was not available for study, so it is unclear whether it normally produces such a peptide. Microorganisms do not readily build resistance to the tridecaptins and, encouragingly, this new compound behaves similarly. Further, the newly identified compound does have complementary bioactivity - acting against several Gram-positive bacteria.

The other notable hit is an antifungal $\mathrm{N}$-acylated nonapeptide, which is encoded by a gene cluster found in the genome of Xenorhabdus nematophila. No structurally similar natural products have been reported, and the peptide is active against several clinically relevant fungal pathogens.

Brady and co-workers have used their new methodology to identify bioactive molecules not available through traditional methods, including those from bacteria not amenable to laboratory culture. "There is more and more genome sequencing data available," says Brady. "Combined with improvements in natural product prediction algorithms, we hope this will lead to the application of this approach to the identification of a growing number of new bioactive small molecules."

Stephen G. Davey

ORIGINAL ARTICLE Vila-Farres, X. et al. Antimicrobials inspired by nonribosomal peptide synthetase gene clusters. J. Am. Chem. Soc. 139 1404-1407 (2017) FURTHER READING Chu, J. et al. Discovery of MRSA active antibiotics using primary sequence from the human microbiome. Nat. Chem. Biol. 12, 1004-1006 (2016) 